\title{
Analysis of left ventricular behaviour in diastole by means of finite element method
}

\author{
C. A. VINSON, D. G. GIBSON, AND A. L. YETTRAM \\ From the Department of Mechanical Engineering, Brunel University, Uxbridge, and the \\ Cardiac Department, Brompton Hospital, London
}

SUMMARY The human left ventricle in diastole can be modelled as a passive structure with incremental internal pressure change being considered as the load. Recent developments in engineering stress

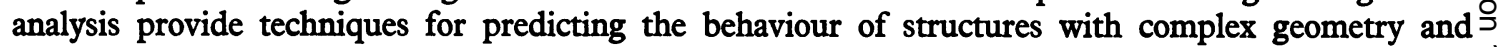
material properties, as is the case with the left ventricle. That which is most appropriate is the finite $\vec{c}$ element method which requires the use of a large digital computer. The ventricles of 2 patients have $\stackrel{0}{5}$ been studied during diastole, the geometries having been derived from cineangiographic data (biplane), and the pressure by means of catheter-tip manometers. Various descriptions of myocardial stress/strain relations have been assumed and applied to the left ventricular wall in order to obtain the best match $\vec{v}$ between the calculated and observed deformation patterns. The manner in which the value and dis- ${ }^{\circ}$ tribution of stiffness in the left ventricle influences the shape change can therefore be determined, and possible clinical implications deduced.

It is generally accepted that abnormalities of ventricular filling may influence overall cardiac function. In the presence of left ventricular hypertrophy, pressure-volume relations are abnormal, and peak rates of diastolic wall movement reduced. Coronary artery disease may cause abnormal wall movement during isovolumic relaxation caused by asynchronous relaxation so that at the time of mitral valve opening, myocardium in non-ischaemic areas may be at or near its end-diastolic position. In order to use these observations of cavity size and configuration to predict the mechanical behaviour of the myocardium, it is necessary to consider relations between wall stress and strain (displacement). Initial attempts to do this were based on the assumption of linear elasticity and unrealistic geometrical models such as spheres or ellipsoids. Since the ventricle is a three-dimensional object with no special planes of symmetry, profiles in at least two planes are needed at each instant, most satisfactorily provided, at present, by biplane angiography. Pressure is reliably measured by a catheter tip manometer, and estimates of wall thickness can be made by echocardiography or cineangiography. If myocardial properties are to be assessed in this way, a comprehensive description must include values for

Received for publication 26 April 1978 the three-dimensional stress for all regions of the wall. The longitudinal and circumferential stresses $\frac{\rho}{3}$ may vary across the wall between endo- and epi- $\bar{T}$ cardium, and also in different parts of the ventricle, particularly when its shape is irregular, while shear stresses are also present in the wall. Further, the $\underset{\mathbb{D}}{\mathbb{D}}$ myocardium itself is not homogeneous, and it has also been known for over three hundred years to 3 have a spiral structure. In addition, the properties of myocardium cannot be described in simple terms. $\frac{\text { ? }}{3}$ It is probably not linearly elastic, may show viscous effects, and values are dependent on the direction in which they are measured. Simple estimates, based $\frac{}{0}$ on Laplace's law and ellipsoidal models do not take account of these considerations, so that their possible $\mathcal{N}$ clinical importance has not been assessed. In order N to circumvent these difficulties, we have used the N finite element technique. The essence of this is that ${ }^{\omega}$ a continuous solid which is acted on by forces or deformations is idealised into an assemblage of separate finite regions or elements. These are taken $\stackrel{\Phi}{+}$ to be interconnected at specific points (nodes) on their common boundaries. Assuming a relation ${ }^{\circ}$ between stress and strain within each element, a set $\stackrel{\mathbb{\Phi}}{\Omega}$ of equations can be derived relating nodal forces to $\frac{\mathbb{1}}{\mathrm{N}}$ nodal displacements. The number of these equations $\cong$ is large, and depends on the fineness of subdivision chosen for the actual solid, the choice being limited 
by the capacity of the computer and computing time which is available. The technique thus allows the interrelations between wall stresses, wall position, and material properties to be investigated. Its use to calculate wall stress requires a detailed knowledge of the material properties of myocardium, which is not at present available. In the present study two cases have been investigated in detail. The method used was to compare observed wall displacement during diastole with that predicted by the finite element method. These predictions depend on the mechanical properties of the wall specified in the modelling procedure, so that the extent to which their alteration affected left ventricular pressurevolume relations, and in particular the agreement between observed and predicted values of wall displacement could be quantified. In this way, it was hoped to get some idea of the possible significance of factors such as the properties of the mitral annulus, the effects of variation in shear modulus or regional myocardial properties or the implication of the fibrous structure of the wall, which cannot be studied using the standard Laplace approach, and yet which may have clinical significance.

\section{Methods}

Two patients were studied in detail. Case $1 \mathrm{had}$ anginal pain, but normal left ventriculogram and coronary arteriograms. Case 2 had a dilated left ventricular cavity, but normal coronary arteriograms, and was assumed to have cardiomyopathy. Haemodynamic results are given in Table 1.

Left ventriculography was performed in posteroanterior and lateral projections at 50 frames/s, with a No. 8 Millar catheter with a micromanometer at its tip. Left ventricular pressure was recorded throughout the angiogram photographically, at a paper speed of $200 \mathrm{~mm} / \mathrm{s}$. Cavity outlines and wall thickness were digitised frame by frame as previously described (Gibson and Brown, 1975).

From these biplane films, a three-dimensional reconstruction of the left ventricle was made. Two templates were constructed for each of the views chosen for analysis, which corresponded with the

Table 1 Haemodynamic values

\begin{tabular}{lcc}
\hline & Case 1 & Case 2 \\
\hline Right atrial pressure $(\mathrm{mmHg})$ & $\overline{3}$ & $\overline{19}$ \\
Right ventricular pressure $(\mathrm{mmHg})$ & $25 / 0,5$ & $57 / 10,19$ \\
Pulmonary artery pressure $(\mathrm{mmHg})$ & $25 / 10 \overline{(13)}$ & $55 / 23 \overline{(30)}$ \\
Left ventricular pressure $(\mathrm{mmHg})$ & $120 ! 5,10$ & $130 / 12,25$ \\
Cardiac index $\left(1 /\right.$ min per $\left.\mathrm{m}^{2}\right)$ & $3 \cdot 6$ & $3 \cdot 0$ \\
Ejection fraction $(\%)$ & 68 & 45 \\
\hline
\end{tabular}

time of minimum left ventricular pressure. These profiles were then transferred to a block of polyurethane, at right angles to one another. The block was then painted, and the corners gradually removed until only 2 lines of paint remained, corresponding to the two biplane outlines. Though this technique clearly contains a subjective element, the resulting models appeared to resemble left ventricular cavity shape, and it was of interest that the septal region was clearly apparent, though not obvious on either of the original cavity outlines. Using these models as templates of the cavity, wall thickness was built up around them using plaster of Paris, to a depth indicated by the wall thickness measurements from the angiograms around the base of the heart, decreasing at the apex to a thickness of $2 \mathrm{~mm}$. The grid for the finite element model was then inscribed by hand, and the three-dimensional co-ordinates of the nodal points measured using a Ferranti 'Conquest' 3D digitiser. ${ }^{1}$

\section{FINITE ELEMENT MODEL}

Thirty-six brick type finite elements were chosen to represent the left ventricle, consisting of 6 layers and 6 elements per layer. The top layer, $1 \mathrm{~mm}$ thick, was used to describe the annulus fibrosus, and the bottom layer, $10 \mathrm{~mm}$ in diameter and $2 \mathrm{~mm}$ thick, to describe the apex (Keith, 1907). The model is illustrated in Fig. 1 and described in detail elsewhere (Yettram and Vinson, 1978a). It was considered to be restrained only in the region of the atria, i.e. along the upper, inner, edge of the annulus fibrosus. Pressure loads were defined from the diastolic pressure record, and were assumed to be uniform throughout the ventricle. Regional variation in pressure has been shown in man (Traill et al., 1978), though only during early diastole. Right ventricular and pericardial pressures were not considered.

The elements of the assemblage were given various combinations of elastic properties reviewed in detail elsewhere (Yettram and Vinson, 1978b). A specimen set of these properties is given in Table 2 . Here the annulus was considered to be isotropic, whereas the myocardium was taken as having a longitudinal stiffness one-half that in the circumferential or cross wall direction. The apex was taken as having properties of collagen. The computer programme used to solve the equations has been described in detail elsewhere (Vinson, 1977), and was run on the University of London CDC computer system and on the Prime 300 system at Brompton Hospital.

${ }^{1}$ By courtesy of RAE, Farnborough. 


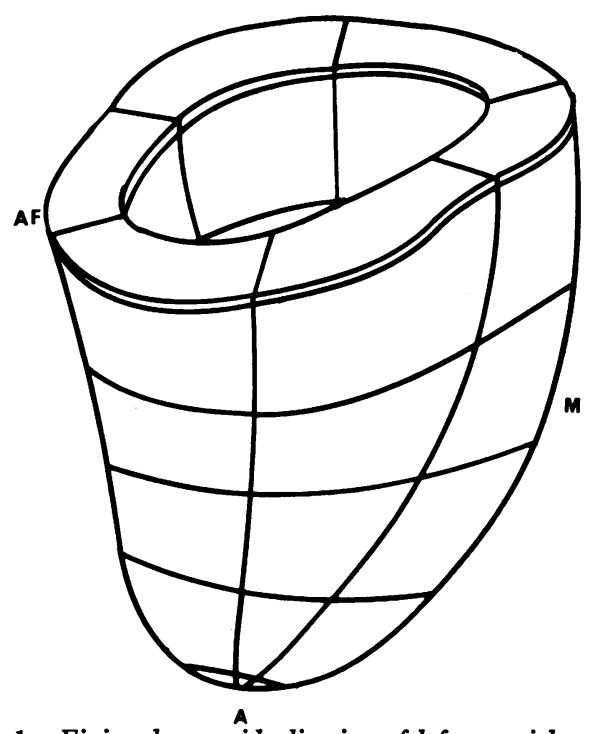

Fig. 1 Finite element idealisation of left ventricle. $M$ : myocardium, $A F$ : annulus fibrosus, $A$ : apex.

Table 2 Specimen set of elastic properties-myocardium

\begin{tabular}{|c|c|c|c|c|}
\hline $\begin{array}{l}\mathbf{E}_{\mathbf{x}} \\
\mathbf{E}_{\mathbf{y}} \\
\mathbf{E}_{\mathbf{z}}\end{array}$ & $\begin{array}{r}8 \\
16 \\
16\end{array}$ & $\begin{array}{ll}G_{x y} & 5 \cdot 41 \\
G_{y z} & 5 \cdot 41 \\
G_{z x} & 5 \cdot 41\end{array}$ & $\begin{array}{ll}\mu_{\mathrm{xy}} & 0.24 \\
\mu_{\mathrm{yz}} & 0.48 \\
\mu_{\mathrm{zx}} & 0.48\end{array}$ & $\begin{array}{ll}\mu_{y x} & 0.48 \\
\mu_{z y} & 0.48 \\
\mu_{x z} & 0.24\end{array}$ \\
\hline
\end{tabular}

E: modulus of elasticity $\left(\mathrm{kN} / \mathrm{m}^{2}\right) ; \mathrm{G}$ : modulus of rigidity $\left(\mathrm{kN} / \mathrm{m}^{2}\right)$; $\mu$ : Poisson's ratio. Material principal directions denoted by $x, y, z$.

\section{PARAMETRIC STUDIES OF LEFT VENTRICULAR} BEHAVIOUR

A number of modifications was then made to the left ventricular wall properties, and the relation between observed and predicted noted. These modifications included:

(1) Alterations in the properties of the annulus fibrosus. This was initially assumed to be a ring of collagenous tissue, with elastic properties as defined in Table 3. Subsequently, it was remodelled as having elasticity similar to that of myocardium.

(2) The apex was initially assumed to consist of fibrous tissue, with a diameter of $1 \mathrm{~cm}$, and so to have properties similar to those of collagen. Comparison was also made with the apex having elasticity varying between that of the myocardium and that for collagen.

(3) Non-homogeneous myocardium. Following a suggestion of Nikravesh (1976), the ventricle was remodelled as being stiffer toward the base than the apex. The values were then reversed, so that the ventricle was stiffer at the apex.

(4) Cross-wall stiffness. Based on the suggestion of Janz and Grimm (1972) the transverse or cross-wall
Table 3 Initial elastic properties-annulus fibrosus

\begin{tabular}{|c|c|c|c|c|c|}
\hline $\left.\begin{array}{l}E_{\mathbf{x}} \\
E_{\mathbf{y}} \\
E_{z}\end{array}\right\} 3.9 \times 10^{8}$ & $\left.\begin{array}{l}\mathbf{G}_{\mathbf{x y}} \\
\mathbf{G}_{\mathrm{yz}} \\
\mathbf{G}_{\mathrm{zx}}\end{array}\right\}$ & $1.573 \times 10^{5}$ & $\left.\begin{array}{l}\mu_{x y} \\
\mu_{y z} \\
\mu_{z x}\end{array}\right\}$ & 0.24 & $\begin{array}{l}\mu_{y x} \\
\mu_{z y} \\
\mu_{x z}\end{array}$ \\
\hline
\end{tabular}

E: modulus of elasticity $\left(\mathrm{kN} / \mathrm{m}^{2}\right) ; \mathrm{G}$ : modulus of rigidity $\left(\mathrm{kN} / \mathrm{m}^{2}\right) ;$; $\mu$ : Poisson's ratio.

elasticity in the outer two-thirds of the ventricular $\overrightarrow{0}$ wall might be one-half the tangential value. Thus $\overrightarrow{-}$ the myocardium in case 1 was modified to have a tangential stiffness of twice that of the transverse stiffness. Shear modulus is an independent variable ${ }_{\perp}^{+}$ and was taken as being unaffected by this manoeuvre. (5) Shear modulus. Shear modulus was then studied, and was reduced from an initial value of $5.41 \mathrm{kN} / \mathrm{m}^{2}$ to 4 and finally $1.52 \mathrm{kN} / \mathrm{m}^{2}$, the last value being that suggested by Janz and Grimm $_{C}^{\vec{C}}$ (1972).

(6) The ratio of longitudinal to circumferential stiffness. This value was reduced to 0.91 derived $₹$ from earlier work using ellipsoidal models $\left(\right.$ Yettram $_{\vec{\varphi}}$ and Vinson, 1978b), from its initial value of 1 . The effects of a further reduction to 0.5 were also studied. (7) Effects of fibre angle. Since fibre angle varies? across the wall from $+60^{\circ}$ to $-60^{\circ}$, its effects could not be investigated in detail using the present model with wall thickness represented by only a singleô element. Nevertheless, if the inner third of the wall $\stackrel{\circ}{\rightrightarrows}$ is more flexible than the remainder (Janz and윽 Grimm, 1972), then there may well be a net effective spiral angle away from the circumferentialo direction. Accordingly, arrangements were examined of spirals at $10^{\circ}$ and $30^{\circ}$, clockwise, from base toळ apex.

(8) Effects of myocardial infarction. Initially, an infarction may be modelled as a region more exten - . sible than surrounding myocardium, but over 2 to윽 4 weeks it becomes replaced by fibrous tissue. Accordingly, both types were modelled, each in-옥 volving 20 to 30 per cent of the total myocardial volume (Parmley et al., 1973). Three sites were chosen, shown in Fig. 2. These included the basal $N$ and apical halves of the free wall, and an anteriorly positioned vertical strip.

Throughout all the above analyses, values for ${ }^{\mathrm{C}}$ wall stress were also derived. Using the generalised? three-dimensional approach, there were 6 stresses? to be considered at any point. These are the 3 directos and 3 shear stresses; more easily interpretable are the maximum principal stress, and the local hydro- $-\overline{0}$ static stress.

\section{Results}

When the left ventricular wall is assumed to have the mechanical properties given in Table 2, then the 


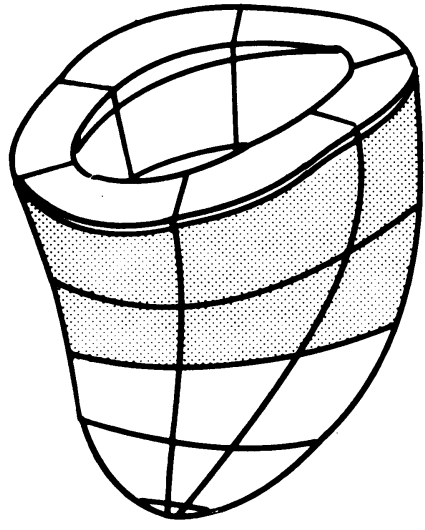

[a]

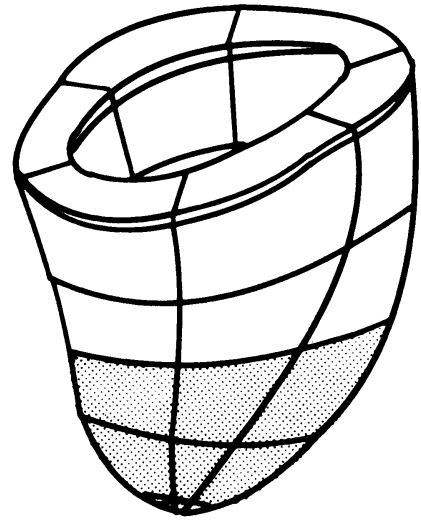

[b]

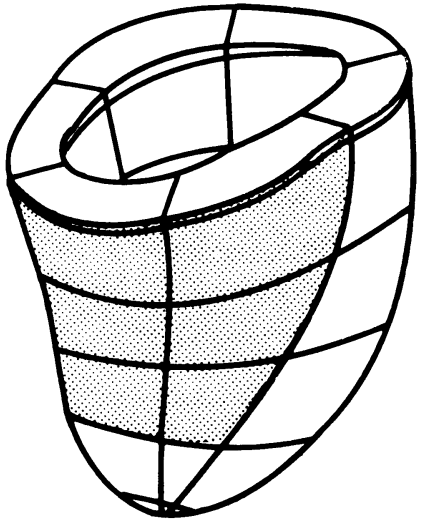

[c]

Fig. 2 Three infarcted regions considered. (a) upper infarct, (b) lower infarct, (c) anterior infarct.

deflection profiles which result are shown in Fig. 3. These are based on the displacements of the nodal points on those mid-plane sections representing the $\mathrm{PA}$ and lateral views. These are compared with the observed outlines of the cavity on the two angiograms. In comparing the two, allowance must be made for the fact that the angiogram does not present a true section but rather an overall profile in any particular projection, so that regions distant from the plotted region may deflect enough to form the boundary as seen on the $x$-ray silhouette. The distributions of the maximum principal stresses/unit internal pressure on the endo- and epicardial surfaces are shown in Fig. 4. The highest values seen in the cases examined appeared to be over the right anterior and left posterior regions of the endo-

Posterior

Anterior
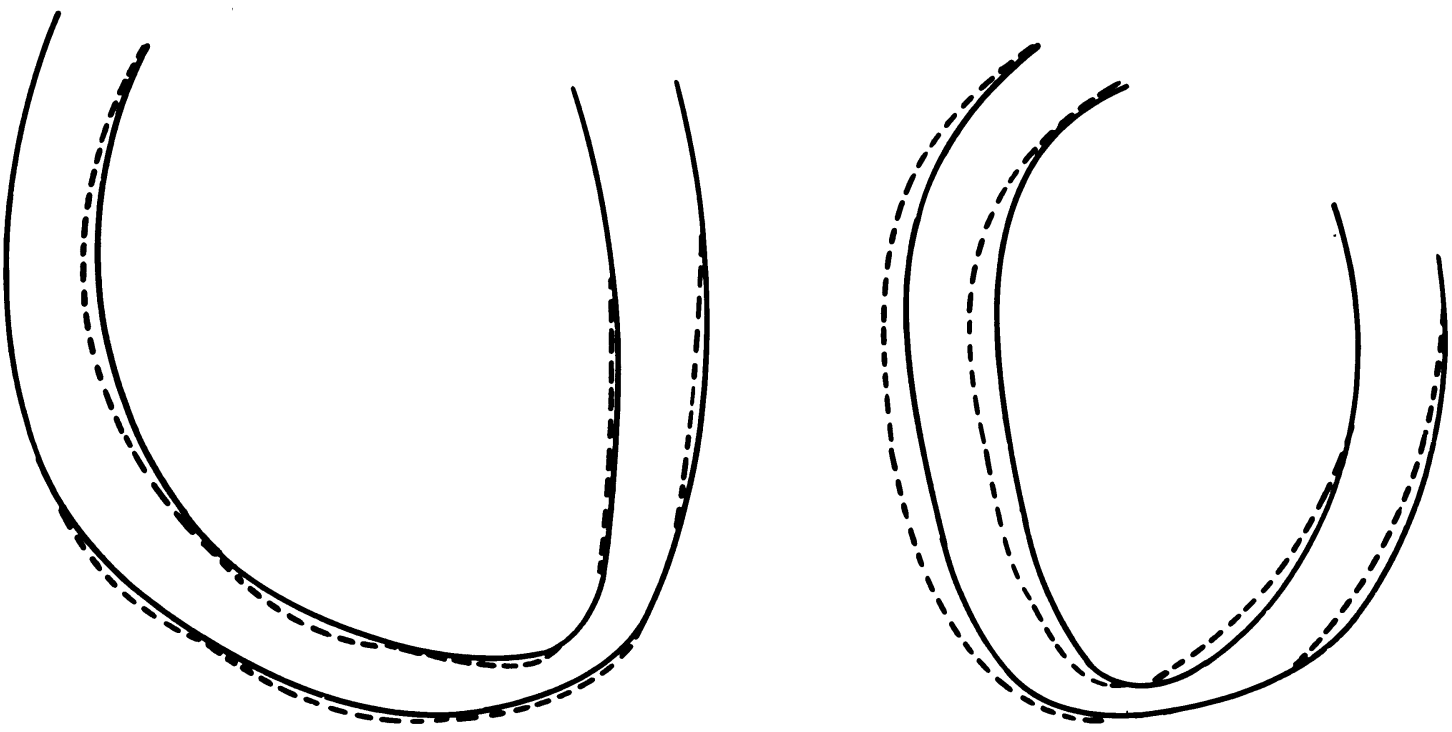

Lateral

A P

Fig. 3 Deflection profiles for myocardium (shown as dashed line) under increment of internal pressure (case 2). 

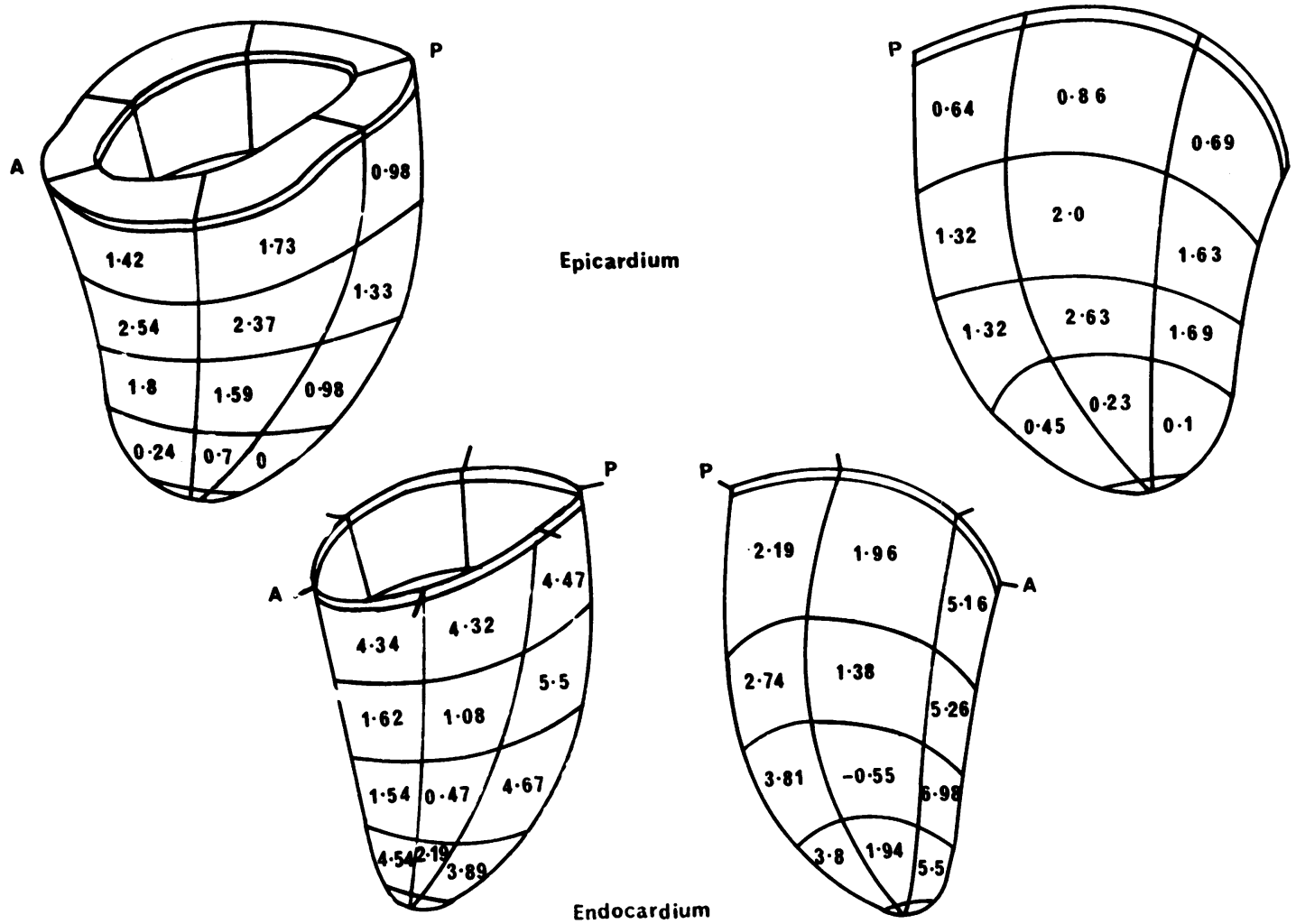

Fig. 4 Patterns of absolute values of principal direct stresses per unit internal pressure over epicardial and endocardial surfaces (case 2).

cardium. These values are of the same order as those obtained by Hamid and Ghista (1974), though these authors observed maximum values towards the apex.

\section{ANNULUS FIBROSUS}

Initially the annulus fibrosus was given the mechanical properties of collagen. When it was remodelled to have the same properties as myocardium, this resulted in improved agreement between observed and predicted values for displacements, in that overall movement of the cavity towards the septum occurs, instead of relative akinesis under the previous conditions. With a completely rigid mitral valve ring, whose outer edge is constrained not to move upwards, the overall increase in volume is less than with a flexible ring, thus rendering the ventricle stiffer.

APEX

When the stiffness of the apex was altered there was no change in the pattern of wall movement. The properties of the apex, therefore, do not appear critical in determining the pattern of wall movement during diastole.
MYOCARDIAL PROPERTIES

In the previous sections, the properties of the myocardium have been assumed to be uniform 0 throughout the left ventricle. It was noted however, in case 1, that inward movement was observed 0 towards the apex, which did not seem compatible $₹$ with any passive model. When the ventricle was re-은 modelled to give non-homogeneous myocardial $\supset$ properties from base to apex in an effort to explain this, agreement between observed and predicted N displacements became less good in both cases. When the base was considered to be stiff, then the ampli- 0 tude of movement was too great at the apex. In $\omega$ case 1 , the ventricle was then remodelled as being stiffer at the apex, but this resulted in deflections ${ }^{\circ}$ that were too large, both in terms of regional wall movement and of overall volume increase. Modifica-? tion of cross-wall stiffness had little effect on pre- $\frac{T}{0}$ dicted displacements, but a reduction in shear ${ }_{\mathbb{\mathrm { D }}}$ modulus from 5.41 to $4.0 \mathrm{kN} / \mathrm{m}^{2}$ caused a pro- $\frac{?}{8}$ nounced increase in the posteroanterior deflections. $\unrhd$ A further reduction to $1.52 \mathrm{kN} / \mathrm{m}^{2}$, as suggested by Janz and Grimm (1972), again increased the overallo deflection in the posteroanterior, with a lesser effecto 
in the lateral. When the ratio of longitudinal to circumferential stiffness was altered from 1.0 to 0.91 , deflections were unaltered. However further reduction to 0.5 produced results that compared well with the angio deflection showing appropriate regional movement though the overall effect was still slightly too great. The high sensitivity to this ratio that has been previously reported by studying ellipsoids (Yettram and Vinson, 1978b) appears to be merely a function of the use of simplistic models, which disappears when a more realistic approach is used. Predicted displacements, however, were very sensitive to the two changes in effective fibre angle of $10^{\circ}$ and $30^{\circ}$. A spiral structure effectively makes the wall less stiff, and significant displacements now appear in the lateral view. The model used therefore suggests that the ratio of circumferential to longitudinal modulus, the variation in fibre angle, and to a lesser extent in shear modulus are important factors in determining displacement during diastole, while the properties of the apex are not. Agreement was not improved by the assumption of regional differences in material properties.

\section{EFFECTS OF MYOCARDIAL INFARCTION}

When the infarction was taken as flexible, with elasticity one-quarter that of the surrounding myocardium, the main effect was local distension, particularly when the infarct involved the basal part of the ventricle. When the infarct was given the properties of collagen, the effects were more complex. The infarcted area appeared rigid, as expected, but in addition the whole ventricle appeared stiffer, particularly when the basal region was involved. This effect was not a generalised one, but was the result of reduced local movement, both at the site of the infarct itself, and also on the opposite wall, in this case, the septum.

\section{Discussion}

There is a considerable body of evidence to suggest that the physical properties of the myocardium are abnormal in left ventricular hypertrophy or coronary artery disease (Mirsky, 1976). Methods have been suggested whereby the material properties of the left ventricular wall, in terms of its elasticity, can be derived from measurements of cavity size, wall thickness, and diastolic pressure. In order to use those methods, based on Laplace's law, however, a number of simplifying assumptions about cavity shape and the nature of the myocardium itself must be made. In the present study we have used the technique of finite element analysis in order to circumvent some of these limitations. Once this more versatile technique is introduced, it immediately becomes apparent that comprehensive estimates of deformation and wall stress require a corresponding knowledge of the material properties of the myocardium. This includes specific information about the apex and mitral annulus, variation in elastic modulus across the wall or in different regions of the cavity, knowledge about shear moduli and fibre angle, and finally consideration of the fibrous structure of the myocardium itself. This information is not necessary when using the Laplace equation, and thus has been little considered in the past.

Finite element methods have been used by a number of authors using a variety of element types. Most previous analyses have assumed the left ventricle to have either a circular or an elliptical cross-section. Gould et al. (1972) used a convenient longitudinal axis passing through the apex of the left ventricular angio, the left and right sides of the chamber were revolved about this axis, and these analysed using shell ring elements. Janz and Grimm (1972) assume the diastolic ventricle to have a circular cross-section and use a ring element for their analysis. Janz et al. (1974) refined this analysis to accommodate non-linear geometric effects. Neckyfarow and Perlman (1976) also assume a circular cross-section but use a thick shell hexahedron. The three-dimensional brick type element was employed by Hamid and Ghista (1974), but they used single plane angio data and assumed an elliptical cross-section having the axis length ratio suggested by Sandler and Dodge (1968). In all these studies values of material properties are estimated from experimental results or from analysis of simpler ellipsoidal models.

Since some indication of the material properties of the myocardium can be obtained from simple elliptical methods, it initially seemed possible that a more comprehensive account might be derived from the use of finite element methods. This approach, described in detail elsewhere (Vinson, 1977), was not fruitful. Though the finite element technique can be adapted for use in this way, it became apparent that the equations were ill defined and needed large amounts of data not readily available and that values of the material properties obtained were critically dependent on the deflections measured.

A different approach was, therefore, used in the present study which was aimed at identifying some of those factors which might be of clinical significance. The initial data were insufficient to obtain a unique set of values of material properties so that estimates were aimed at by an iterative process and comparison with the angiograms. The estimates are thus consistent with the angio data, but not necessarily unique. Using these as a basis we have ex- 
plored the effects of changes in these initial material properties on the predicted angiographic outlines. The results of studies of this type are not directly applicable to the individual patients. Instead, they indicate that when structures whose shape approximates to that of an actual left ventricle rather than to some idealised geometrical figure are considered to operate over observed ranges of diastolic pressure, then variation of diastolic wall properties within a range that has some experimental basis causes changes in wall deflection that can be measured using standard clinical methods. In the present study, we have attempted to identify sources of variation that produce the most significant alterations in these deflections. The results must not be equated with experimental observation, but can, perhaps, be used to suggest areas in which more direct measurement is likely to be fruitful in understanding cardiac function.

The procedure described above leads to a number of conclusions of potential clinical significance. The mitral annulus appears to have an appreciable influence on left ventricular diastolic wall movement. This may have practical importance after mitral valve replacement. At present, a variety of mechanical prostheses or heterografts are used, but all these have in common is a rigid mitral valve ring. $A b-$ normal left ventricular wall movement has previously been documented after mitral valve replacement (Miller et al., 1973; Sutton et al., 1977): septal movement is reversed and the peak rate of increase of left ventricular dimension is reduced. This abnormal pattern of filling is not seen should a paraprosthetic leak develop, and it does not occur after mitral valve repair, when the ring is supported by two discontinuous sutures. Though changes in the diameter of the mitral valve ring have not been reported in man, they have been described in postmortem specimens arrested at different stages of the cardiac cycle by Lian (1909). If a mitral prosthesis could be designed without a rigid ring, it might have significant haemodynamic advantages.

The material properties of the myocardium are complex. Three of these may merit further study in view of their possible effect in determining left ventricular wall position during diastole: the degree of anisotropy, variation in shear modulus, and in particular the spiral arrangement of the muscle fibres. These factors probably reflect the high degree of organisation of the normal left ventricle, which has been little studied in man owing to the difficulty in gaining information in vivo. Nevertheless, its effects on left ventricular function appear so profound that conclusions based on models in which the myocardium is assumed to be homogeneous and isotropic are seen to be of limited value, and further attempts to supply the necessary information appear essential. It is also apparent that the stress $\stackrel{5}{+}$ pattern within the myocardium is complex, and that $\frac{}{2}$ it is not possible to use a single cross-section to $\frac{\bar{F}}{\bar{D}}$ describe it. This is of significance in attempting to $\frac{\widehat{D}}{\widehat{D}}$ derive material properties in vivo, as has previously $\cong$ been pointed out, and also in attempting to under- $\%$ stand the very much more difficult problem of $\vec{\circ}$ describing systolic events.

The conclusions regarding myocardial infarcts $\vec{\omega}$ may also have clinical significance, particularly those $\frac{\rho}{\partial}$ dealing with fibrous scars. Previous observations

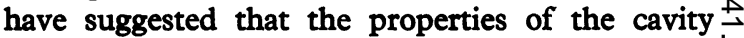
may change after myocardial infarction, with an increase in stiffness (Bristow et al., 1970), as pre- ${ }^{\circ}$ dicted by the present results. It also appears that the $\frac{\rho}{2}$ physiological effects of an infarct depend not only $\overrightarrow{ }$ on its size and intrinsic properties, but also on its site within the ventricle. The disturbance to filling appears to be greater when the basal region is involved than when the infarct is apical. It should $\overrightarrow{0}$ be possible to investigate this difference clinically. Finally, the results suggest that a localised region of ${ }^{\circ}$ abnormal wall properties need not necessarily lead to an abnormality of wall movement confined to the same site, but that such disturbances may be more $\bar{D}$ generalised, affecting wall movement elsewhere in

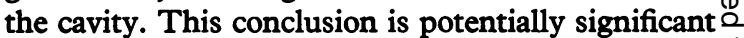
if attempts are made to localise abnormalities of wall $\overrightarrow{\overrightarrow{0}}$ movement in patients with single vessel coronary 3 artery disease. The present results suggest that this assumption might be premature.

This study has many limitations. The possible effects of right ventricular pressure, pericardial $\stackrel{D}{\rightleftharpoons}$ pressure, or the mitral subvalve apparatus on left $\overline{0}$ ventricular wall movement were not investigated. Angiography is not a wholly satisfactory method in $ᄋ$ defining end-systolic wall movement, and a comprehensive description of left ventricular wall posi-응 tion cannot be obtained from views in only two $>$ planes. Measurements of wall thickness were limited to a small region on each cine frame. The interventricular septum almost certainly has a structure $\sigma$ different from that of free wall, and should be $\mathcal{N}$ modelled separately. It is possible that two- $\omega$ dimensional echocardiographic methods might be useful as a supplement to angiography, particularlyo to gain a more comprehensive description of the $₫$ distribution of wall thickness, while, in the more $\stackrel{\text { ? }}{+}$ distant future, computerised tomography or related $\underset{T}{T}$ methods may be of value.

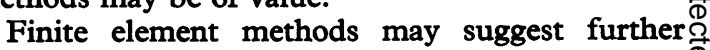
possibilities of clinical significance. At present, $\varrho$ their use has significant limitations in routine clinicalo practice, particularly in respect to the relatively 0 limited amount of information available to construct: 
the model, and the amount of computation involved. In spite of this, however, they may be helpful in exploring the implications of myocardial structure and properties on overall left ventricular function, and in giving some insight into the relation between the two which cannot easily be appreciated when simpler methods are used.

We acknowledge with thanks a generous grant from May and Baker towards computing equipment.

\section{References}

Bristow, D., Van Zel, B. E., and Judkins, M. P. (1970). Systolic and diastolic abnormalities of the L.V. in coronary artery disease: studies in patients with little or no enlargement of ventricular volume. Circulation, 42, 219-228.

Gibson, D. G., and Brown, D. J. (1975). Continuous assessment of left ventricular shape in man. British Heart fournal, 37, 904-910.

Gould, P., Ghista, D. N., Brombolich, L., and Mirsky, I. (1972). In vivo stresses in the human left ventricular wall: analysis accounting for the irregular 3-dimensional geometry and comparison with idealised geometry analyses. fournal of Biomechanics, 5, 521-539.

Hamid, M. S., and Ghista, D. N. (1974). Finite element analysis of human cardiac structures. In Finite Element Methods in Engineering, pp. 337-348. University of New South Wales.

Janz, R. F., and Grimm, A. F. (1972). Finite element model for the mechanical behaviour of the left ventricle. Circulation Research, 30, 244-252.

Janz, R. F., Kubert, B. R., and Moriarty, T. F. (1974). Deformation of diastolic left ventricle-II. Non-linear geometric effects. Fournal of Biomechanics, 7, 509-516.

Keith, A. (1907). An account of the structures concerned in the production of the jugular pulse. Fournal of Anatomy and Physiology, 42, 1-25.

Lian, C. (1909). Contribution à l'étude de Ja physiologie de l'appareil valvulaire mitral. Fournal de Physiologie et de Pathologie Générale, 11, 597-612.

Miller, H. C., Gibson, D., and Stephens, J. (1973). The role of echocardiography and phonocardiography in the diagnosis of mitral paraprosthetic regurgitation with StarrEdwards prosthesis. British Heart Fournal, 35, 1217-1225.

Mirsky, I. (1976). Assessment of passive elastic stiffness of cardiac muscle: mathematical concepts, physiologic and clinical considerations, directions of future research. Progress in Cardiovascular Diseases, 18, 277-308.

Neckyfarow, C. W., and Perlman, A. B. (1976). Deformation of the human left ventricle: material and geometric effects. In Proceedings of the 4th New England Bioengineering Conference, pp. 169-172. Ed. by S. Saha. Pergamon, New York.

Nikravesh, E. P. (1976). Optimization in finite element analysis with special reference to three dimensional left ventricular dynamics. D.Eng. Thesis, Department of Electrical Engineering, Tulane University, New Orleans, Louisiana.

Parmley, W. W., Chuck, L., Matloff, J. M., and Swan, W. J. C. (1973). In vitro length-tension relations of human ventricular aneurysms. American fournal of Cardiology, 32, 889-894.

Sandler, H., and Dodge, H. T. (1968). The use of single plane angiocardiograms for the calculation of left ventricular volume in man. American Heart fournal, 75, 325-334.

Sutton, M. G., St.J., Traill, T. A., Ghafour, S. A., Brown, D. J., and Gibson, D. G. (1977). Echocardiographic assessment of $\mathbf{L}$.V. filling after mitral valve surgery. British Heart fournal, 39, 1283-1291.

Traill, T. A., Gibson, D. G., and Brown, D. J. (1978). Intracavity pressure gradients during left ventricular filling: echocardiographic study. Transactions of the European Society of Cardiology, 1, 24.

Vinson, C. A. (1977). Analysis of stress and deformation in the human left ventricle. PhD. Thesis, Department of Mechanical Engineering, Brunel University, Uxbridge, Middlesex, England.

Yettram, A. L., and Vinson, C. A. (1978a). Geometric modelling of the human left ventricle. Submitted for publication.

Yettram, A. L., and Vinson, C. A. (1978b). Orthotropic elastic moduli for LV mechanical behaviour. Medical and Biological Engineering and Computing. In the press.

Requests for reprints to Dr D. G. Gibson, Cardiac Department, Brompton Hospital, London SW36HP. 